\title{
Comparison of Post-Stroke Functional Recovery between Ischemic and Hemorrhagic Stroke Patients: A Prospective Cohort Study
}

\author{
Sabrina Anggi Lubis, ${ }^{1}$ Novitri, ${ }^{2}$ Ahmad Rizal ${ }^{3}$ \\ ${ }^{1}$ Faculty of Medicine Universitas Padjadjaran, ${ }^{2}$ Department of Physical Medicine and \\ Rehabilitation/Dr. Hasan Sadikin General Hospital Bandung, ${ }^{3}$ Department of Neurology Faculty \\ of Medicine Universitas Padjadjaran/Dr. Hasan Sadikin General Hospital Bandung
}

\begin{abstract}
Background: Stroke is differentiated into two types, hemorrhagic and ischemic stroke. Different pathophysiologic mechanism that underlies each stroke type may give different outcome in post-stroke patients. This study aimed to compare the improvement of functional outcome between both types of stroke among stroke patients admitted to Dr. Hasan Sadikin General Hospital (RSHS).

Methods: Consecutive sampling was carried out on first-ever stroke patients admitted to neurological inpatient unit of RSHS from September 2015 to October 2015. Functional recovery, measured by subtracting Canadian Neurological Scale (CNS) on day-8 and day-1 of admission, was compared among two subgroups and analyzed using Mann-Whitney U test. Baseline characteristics were collected and analyzed.

Results: Total of thirteen stroke patients was included in this study: ten ischemic stroke and three hemorrhagic stroke patients. CNS score improvement between ischemic and hemorrhagic stroke patients was not significantly different. However, hemorrhagic stroke had higher score than ischemic stroke with 0.30 CNS score difference.
\end{abstract}

Conclusions: CNS improvement between ischemic and hemorrhagic stroke was not significantly different.

Keywords: Functional recovery, hemorrhagic stroke, ischemic stroke

\section{Introduction}

Stroke causes significant health problems in Indonesia since its survivors develop disability and Indonesia mean age of stroke is 58.8, which is still in productive age. ${ }^{1}$ There are two main types of stroke: (1) ischemic stroke, which is caused by reduction of cerebral blood flow, eventually leading to cerebral infarction and (2) hemorrhagic stroke, which is caused by rupture of blood vessel in the brain, increasing intracranial pressure. ${ }^{2}$

There are differences in term of mortality, severity, and prognosis between ischemic and hemorrhagic strokes. Hemorrhagic stroke is associated with higher mortality and worse functional impairment after stroke. ${ }^{3-5}$ In term of recovery, earlier studies have found that after rehabilitation, hemorrhagic stroke is associated with better functional recovery than ischemic stroke. ${ }^{6-8}$ However, there are many important factors other than stroke type that need to be considered in term of stroke prognosis. $^{2}$

This study was somewhat different from earlier studies that assessed patient's capability of recovery within short duration and without rehabilitation exposure. Thus, it was expected that this study could compare post-stroke functional recovery between ischemic and hemorrhagic stroke patients without many interfering factors.

\section{Methods}

This was a prospective cohort study that consecutively recruited stroke patients in neurological inpatient unit of Dr. Hasan Sadikin General Hospital (RSHS) from September 2015 to October 2015. Patients would be included in this study if they met all the inclusion criteria. Inclusion criteria were: first-ever ischemic or hemorrhagic stroke patients confirmed by CT scan, aged $\geq 18$ years old, signed the informed consent, Glasgow Coma Scale (GCS) $\geq 13$, and onset to admission interval $\leq 48$ hours.

Correspondence: Sabrina Anggi Lubis, Faculty of Medicine, Universitas Padjadjaran, Jalan Raya Bandung-Sumedang Km.21, Jatinangor, Sumedang, Indonesia, Phone: +62 85750280918 Email: anggisabrina18@gmail.com 
Patients who had recurrent stroke attacks or died during the study were excluded. A total of 15 patients were included in this study.

This study used the American Heart Association/American Stroke Association definition of stroke. Ischemic stroke is defined as, "An episode of neurological dysfunction caused by focal cerebral, spinal, or retinal infarction", whereas hemorrhagic stroke is defined as, "A focal collection of blood within the brain parenchyma or ventricular system that is not caused by trauma". ${ }^{9}$ The stroke was classified as ischemic or hemorrhagic using findings on CT scan. Baseline characteristics were collected from medical record and tested for normality using Shapiro-Wilk test. Then the baseline characteristics were analyzed using independent T-Test and Chi Square Test.

Functional recovery was measured using Canadian Neurological Scale (CNS), a simple and standardized tool to assess neurological status. CNS was measured twice, (1) first, at the time of admission in neurological inpatient unit and (2) second, seven days from the first CNS measurement. CNS improvement was calculated by subtracting first CNS score from second CNS score. Shapiro-Wilk test was used to test normality of the data. CNS improvement data was analyzed using Mann-Whitney U test to see the significance of the difference among two subgroups.

Ethics committee of RSHS has reviewed and approved this study through ethical clearance Number: LB.04.01/A05/EC/242/VII/2015.

\section{Results}

During data collection period, there were 55 stroke patients were admitted to neurological inpatient unit of RSHS. Forty patients could not be included in the study either because they had recurrent stroke attacks or had GCS score $<13$. Fifteen stroke patients were eligible for the study consisted of ten ischemic stroke patients and five hemorrhagic stroke patients. Two hemorrhagic stroke patients were further excluded because they had died during data collection.

Complete baseline characteristics among two subgroups are presented on Table 1 . Baseline characteristics among two subgroups were not significantly different.

Data of CNS improvement was not normally distributed. From Mann-Whitney U test result, CNS improvement between ischemic and hemorrhagic stroke was not significantly different (Table 2). However, hemorrhagic stroke had a higher score with 0.30 CNS score improvement difference (Table 2).

\section{Discussion}

Ischemic and hemorrhagic stroke have different mechanism of neuronal injury. In

Table 1 Comparison of Baseline Characteristics among Two Subgroups

\begin{tabular}{|c|c|c|c|c|}
\hline & $\begin{array}{c}\text { Ischemic Stroke } \\
\qquad(\mathrm{n}=10)\end{array}$ & $\begin{array}{c}\text { Hemorrhagic } \\
\text { Stroke } \\
(n=3)\end{array}$ & $\begin{array}{l}\text { Total } \\
(n=13)\end{array}$ & $\mathbf{p}$ \\
\hline Age (year), mean $\pm \mathrm{SD}^{*}$ & $53.70+14.25$ & $58.00+17.06$ & $54.69+14.30$ & 0.668 \\
\hline Male, $\%$ & 70 & 100 & 76.92 & 0.528 \\
\hline $\begin{array}{l}\text { CNS** score at admission, mean } \\
\pm \mathrm{SD}^{*}\end{array}$ & $6.75 \pm 3.97$ & $6.50 \pm 1.80$ & $6.69 \pm 3.52$ & 0.920 \\
\hline Hypertension, \% & 80 & 100 & 84.62 & 1.000 \\
\hline Heart Disease, \% & 40 & 0 & 30.77 & 0.497 \\
\hline Diabetes Mellitus, \% & 10 & 0 & 7.69 & 1.000 \\
\hline
\end{tabular}

Note: ${ }^{*} \mathrm{SD}=$ standard deviation, ${ }^{* *} \mathrm{CNS}=$ Canadian Neurological Scale

Table 2 Comparison of CNS Improvement among Two Subgroups

\begin{tabular}{|c|c|c|c|}
\hline & $\begin{array}{l}\text { Ischemic Stroke } \\
\qquad(\mathrm{n}=10)\end{array}$ & $\begin{array}{c}\text { Hemorrhagic } \\
\text { Stroke } \\
(n=3)\end{array}$ & $\mathbf{p}$ \\
\hline CNS* score improvement, median (range) & $0.70(-3-4.5)$ & $1.00(0-1.5)$ & 0.692 \\
\hline
\end{tabular}




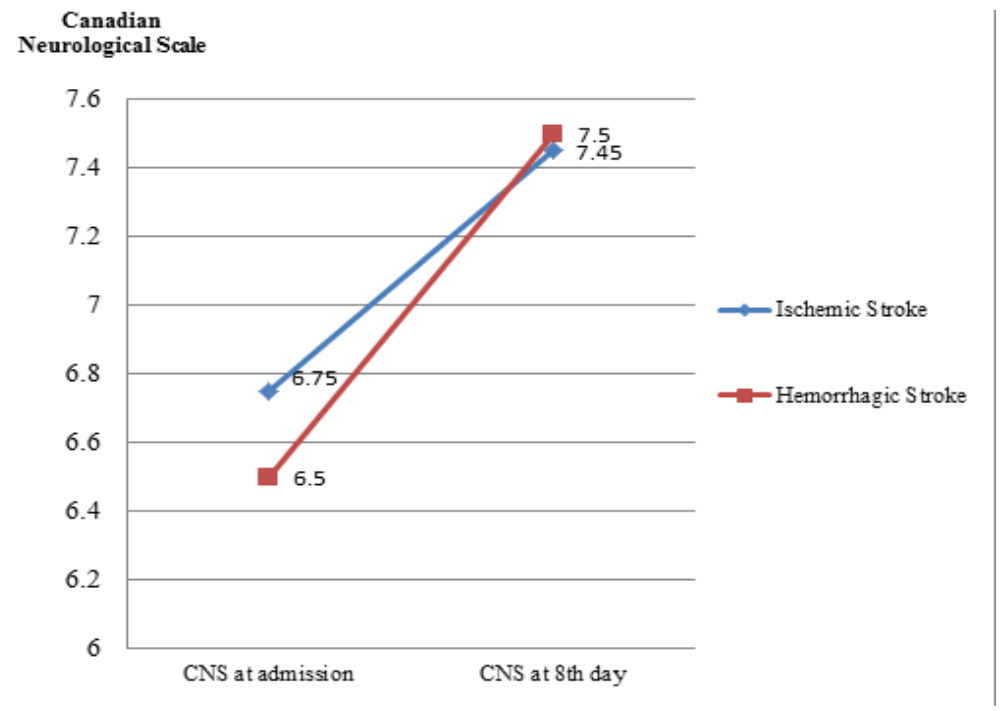

Figure 1 CNS Scores Improvement over Time

ischemic stroke, neuronal injury is caused by hypo-perfusion to the brain and thus irreversible ischemic necrosis is inevitable, whereas in hemorrhagic stroke, neuronal injury is due to the pressure given by intracranial bleeding. Moreover, hematoma that is built in hemorrhagic stroke can cut off connecting pathways in the brain. In ischemic stroke, necrotic neuron persists despite the reperfusion that occurs, but, in hemorrhagic stroke, after blood is drained and intracranial pressure is relieved, neurons may recover.2 Therefore, prognosis of hemorrhagic stroke patients is largely influenced by duration of bleeding and size of hematoma. ${ }^{7,10,11}$ Although hemorrhagic stroke has higher mortality and worse functional outcome than ischemic stroke, $^{3-5}$ hemorrhagic stroke patients who survive have better functional improvement than ischemic stroke. ${ }^{6-8}$

From the result of this study, hemorrhagic stroke patients tended to be more severe on admission, yet they improved faster and had better final CNS scores (Figure 1). Though there was no significant CNS score improvement difference among the two subgroups, this study did found that hemorrhagic stroke patients had higher CNS score improvement than ischemic stroke patients. These findings were consistent with earlier reports that have demonstrated that hemorrhagic stroke is worse at first but significantly better in term of functional recovery than ischemic stroke. ${ }^{3,6-8}$

Gender, age, hypertension, diabetesmellitus, and heart disease, which were considered possible confounding factors in this study, had been controlled. Patients in this study were not in rehabilitation unit, so it can be assumed that there were no compensatory influences to the patient's functional improvement. However, there were several limitations in this study. This study was conducted in RSHS, which is a tertiary health care facility. Thus, patients admitted to this hospital tend to be in severe condition, which was either recurrent stroke patients or stroke patients with GCS score $<13$. Furthermore, this study was conducted only for two months, resulting in small sample size. That sample became the major limitation to this study.

Due to its higher mortality and lower incidence than ischemic stroke, there was disparity in the number of sample among two subgroups. ${ }^{3,5,12}$ Hemorrhagic stroke patients in this study consisted of only three patients, so those samples might not be representative of total hemorrhagic stroke population. This might be the reasonthis study did not reach significant statistical difference.

Therefore, this study concludes that CNS improvement between ischemic and hemorrhagic stroke was not significantly different. Suggestions for future study is to conduct a research in secondary health care facility where stroke patients admitted tend to be in less severe condition. Besides, it is also suggested to do a research in a longer time thus the sample size could be bigger. 


\section{References}

1. Kusuma Y, Venketasubramanian N, Kiemas L, Misbach J. Burden of stroke in Indonesia. Int J Stroke. 2009;4(5):379-80.

2. Caplan LR. Caplan's stroke: a clinical approach. $4^{\text {th }}$ ed. Philadelphia: Elsevier Health Sciences; 2009.

3. Andersen KK, Olsen TS, Dehlendorff C, Kammersgaard LP. Hemorrhagic and ischemic strokes compared stroke severity, mortality, and risk factors. Stroke. 2009;40(6):2068-72.

4. McGuire AJ, Raikou M, Whittle I, Christensen MC. Long-term mortality, morbidity and hospital care following intracerebral hemorrhage: an 11-Year cohort study. Cerebrovasc Dis. 2006;23(23):221-8.

5. Christensen MC, Munro V. Ischemic stroke and intracerebral hemorrhage: the latest evidence on mortality, readmissions and hospital costs from Scotland. Neuroepidemiology. 2008;30(4):239-46.

6. Schepers VP, Ketelaar M, Visser-Meily AJ, de Groot V, Twisk JW, Lindeman E. Functional recovery differs between ischaemic and haemorrhagic stroke patients. J Rehabil Med. 2008;40(6):487-9.

7. Joa K-L, Han T-R, Pyun S-B, Rah U-W, Park J-H, Kim Y-H, et al. Inpatient stroke rehabilitation outcomes in Korea derived from the Korean brain rehabilitation centers' online database system for the years 2007 to 2011. J Korean Med Sci. 2015;30(5):644-50.

8. Bhalla A, Wang Y, Rudd A, Wolfe CD. Differences in outcome and predictors between ischemic and intracerebral hemorrhage the South London stroke register. Stroke. 2013;44(8):2174-81.

9. Sacco RL, Kasner SE, Broderick JP, Caplan LR, Culebras A, Elkind MS, et al. An updated definition of stroke for the 21st Century: a statement for healthcare professionals from The American Heart Association/ American Stroke Association. Stroke. 2013;44(7):2064-89.

10. Mondal RN BS, Islam MJ, Jahan SMS, Alam ABMM, Hussain MM, et al. Short-term predictors of mortality among patients with hemorrhagic stroke. World Heart J. 2014;6(4):273-81.

11. Wang C-W, Liu Y-J, Lee Y-H, Hueng D-Y, Fan H-C, Yang F-C, et al. Hematoma shape, hematoma size, glasgow coma scale score and ICH score: which predicts the 30day mortality better for intracerebral hematoma? PLoS One. 2014;9(7):e102326.

12. Go AS, Mozaffarian D, Roger VL, Benjamin EJ, Berry JD, Borden WB, et al. Heart disease and stroke statistics--2013 update: a report from the American Heart Association. Circulation. 2013;127(1):e6e245. 